\title{
Response to
}

\section{measles outbreaks}

\section{in measles mortality}

\section{reduction settings}

Immunization, Vaccines and Biologicals 


\section{Response to}

\section{measles outbreaks}

in measles mortality reduction settings

Immunization, Vaccines and Biologicals

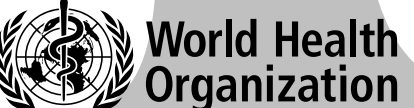




\section{The Department of Immunization, Vaccines and Biologicals thanks the donors whose unspecified financial support has made the production of this document possible.}

This document was produced by the Expanded Programme on Immunization of the Department of Immunization, Vaccines and Biologicals

Ordering code: $\mathrm{WHO} / \mathrm{IVB} / 09.03$

Printed: March 2009

This publication is available on the Internet at: www.who.int/vaccines-documents/

Copies of this document as well as additional materials on immunization, vaccines and biologicals may be requested from:

World Health Organization

Department of Immunization, Vaccines and Biologicals

CH-1211 Geneva 27, Switzerland

- Fax: + 41227914227 •Email: vaccines@who.int

(C) World Health Organization 2009

All rights reserved. Publications of the World Health Organization can be obtained from WHO Press, World Health Organization, 20 Avenue Appia, 1211 Geneva 27, Switzerland (tel: +41 22791 3264; fax: +41 22791 4857; email: bookorders@who.int). Requests for permission to reproduce or translate WHO publications - whether for sale or for noncommercial distribution - should be addressed to WHO Press, at the above address (fax: +41 22791 4806; email: permissions@who.int).

The designations employed and the presentation of the material in this publication do not imply the expression of any opinion whatsoever on the part of the World Health Organization concerning the legal status of any country, territory, city or area or of its authorities, or concerning the delimitation of its frontiers or boundaries. Dotted lines on maps represent approximate border lines for which there may not yet be full agreement.

The mention of specific companies or of certain manufacturers' products does not imply that they are endorsed or recommended by the World Health Organization in preference to others of a similar nature that are not mentioned. Errors and omissions excepted, the names of proprietary products are distinguished by initial capital letters.

All reasonable precautions have been taken by the World Health Organization to verify the information contained in this publication. However, the published material is being distributed without warranty of any kind, either expressed or implied. The responsibility for the interpretation and use of the material lies with the reader. In no event shall the World Health Organization be liable for damages arising from its use.

The named authors alone are responsible for the views expressed in this publication.

Printed by the WHO Document Production Services, Geneva, Switzerland 


\section{Contents}

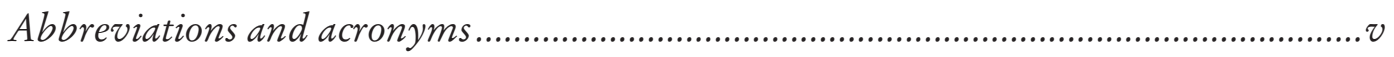

Acknowledgements...............................................................................................

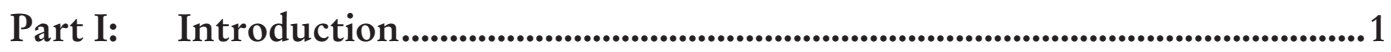

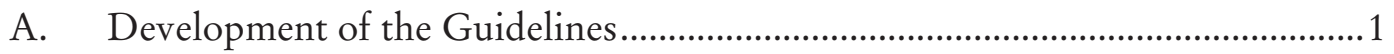

B. Rationale and public-health importance .................................................................

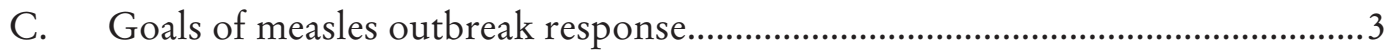

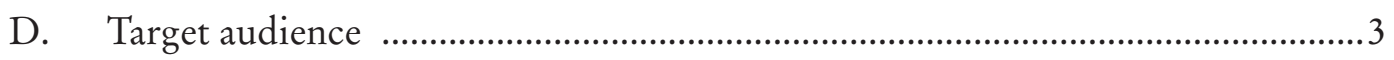

E. Strategies for measles prevention and control: current recommendations ..........3

F. Rationale for use of measles vaccine to control outbreaks.................................... 4

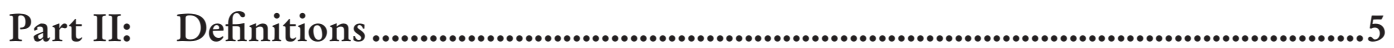

A. Case definitions for measles surveillance and outbreak investigation .................5

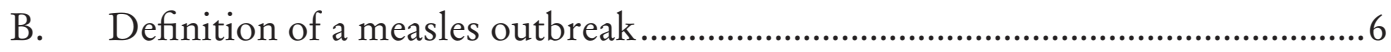

Part III: Confirming, investigating and managing an outbreak .............................8

A. Laboratory confirmation of a measles outbreak ................................................. 10

B. Ensuring adequate clinical case management .................................................... 10

C. Intensifying surveillance and notification .........................................................11

D. Assessing the risk of a large outbreak with high morbidity and

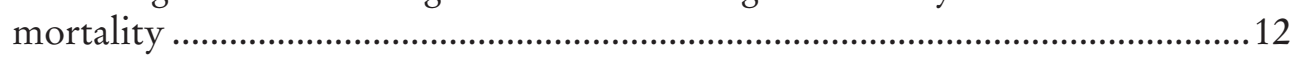

E. Investigating a confirmed measles outbreak.......................................................... 14

F. Implementing control and preventive measures ................................................17

G. Ensuring effective community involvement and public awareness...................22

Part IV: Learning lessons from the outbreak .....................................................23

Annex 1: Laboratory procedures......................................................................................................24

Annex 2: The outbreak coordination committee .......................................................26

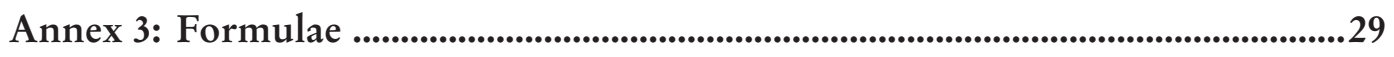

Annex 4: Generic line-list for use during measles outbreaks ...................................31

Annex 5: References ............................................................................................................................33 


\section{Abbreviations and acronyms}

AD

AFRO

AMRO

AR

ARU

ARV

CDC

CFR

EMRO

EPI

EURO

Ig

IHR

IMCI

IU

MSF

NGO

OPD

PCV

PHEIC

PPV

SEARO

SIA

UNICEF

VE

WPRO auto-disable (syringe)

WHO Regional Office for Africa

WHO Regional Office for the Americas

attack rate

attack rates among unvaccinated persons

attack rates among vaccinated persons

Centers for Disease Control and Prevention of the United States Health and Human Services Department

case-fatality ratio

WHO Regional Office for the Eastern Mediterranean

Expanded Programme on Immunization

WHO Regional Office for Europe

immunoglobulin (IgM)

International Health Regulations

Integrated Management of Childhood Illnesses

International Unit

Médecins Sans Frontières

non-governmental organization

outpatient department

percentages of cases in vaccinated persons

Public Health Emergency of International Concern

percentage of the population vaccinated

WHO Regional Office for South-East Asia

supplementary immunization activity

United Nations Children's Fund

vaccine effectiveness

WHO Regional Office for the Western Pacific 


\section{Acknowledgements}

This document has been jointly prepared ${ }^{1}$ by the Departments of Immunization, Vaccines and Biologicals (Dr Alya Dabbagh and Dr Peter Strebel) and Epidemic and Pandemic Alert and Response (Dr William Perea) at the World Health Organization in consultation with the Centers for Disease Control and Prevention of the United States Health and Human Services Department (Dr Vance Dietz and Dr Lisa Cairns), the United Nations Children's Fund (Dr Edward Hoekstra), the Médecins Sans Frontières (Dr Florence Fermon), and Epicentre (Dr Rebecca Freeman-Grais).

Recommended date for updating this document is 2013.

Questions or comments concerning this document should be directed to:

The Department of Immunization, Vaccines and Biologicals in the Family and Community Health Cluster (FCH),

World Health Organization, 1211 Geneva 27, Switzerland.

1 No conflict of interest has been declared by any of the contributors to the document 


\section{Part I: \\ Introduction}

\section{A. Development of the Guidelines}

Current WHO guidance on measles outbreak response published in 1999 (1), emphasized that most outbreaks were either detected too late or spread too rapidly to allow for an effective immunization response. This recommendation was based primarily on a literature review of manuscripts published on the impact of immunization control activities on measles outbreaks in middle- and low-income countries from 1977 to 1993 (2). The validity of these recommendations was reviewed given recent evidence (published and from filed experience) on the impact of outbreak response immunization on measles outbreaks. This review of the evidence was carried out as follows:

- A consultation meeting was organized by WHO in 2006 to discuss the need for updated guidelines and to review the new evidence which supports the guidance provided in the updated document. The meeting was attended by experts from the CDC, Epicentre, MSF Belgium and MSF France, WHO and UNICEF.

- An extensive literature review of data (published from 1995-2006) to re-assess the impact of immunization response to measles outbreaks was carried out and the results were summarized and submitted for publication. This included a review of 277 articles. The key findings of this review are summarized in section F below.

- Review of data from the field and from unpublished studies on the duration of measles outbreaks and effectiveness of interventions with non-selective vaccination.

Based on the initial consultations, a draft document was prepared by the Departments of Immunization, Vaccines and Biologicals and Epidemic and Pandemic Alert and Response and comments from experts were solicited. Experts were from, the CDC, the 6 WHO regional offices, MSF, Epicentre, UNICEF and from country level immunization staff and partners. 


\section{B. Rationale and public-health importance}

Measles is highly contagious and remains a leading cause of childhood mortality. In the pre-vaccine era an estimated 130 million cases occurred each year. At the World Health Assembly in 2005, an ambitious global goal for measles control was established as part of the Global Immunization Vision and Strategy document (3) - that is, to achieve a $90 \%$ reduction in measles mortality by 2010 compared with 2000 .

As of 2008, four of the six WHO regional offices have a measles elimination goal (AMRO, EURO, EMRO, WPRO) and two have a mortality reduction goal (AFRO, SEARO). It is in these latter two regions where most of the global measles deaths occur. Worldwide, since the year 2000, the estimated number of measles deaths has been reduced by $74 \%$. However, despite this remarkable progress in controlling measles, there were an estimated 197000 deaths in 2007 (4).

However, during the past years, three important changes have occurred which necessitate the revision of the recommendation of the above mentioned $1999 \mathrm{WHO}$ document:

- The expanded use of a second opportunity for measles immunization through nationwide mass-vaccination campaigns in high burden countries has resulted in marked reductions in measles incidence associated with reduced community acceptance of large outbreaks.

- Endorsement of the International Health Regulations (2005) highlighted the importance of timely detection and response to events that are of potential international public- health concern (5).

- Recent literature on the impact of outbreak response immunization found measles epidemics in endemic, pre catch-up supplementary immunization activity (SIA) settings can last 3-9 months providing adequate time to mount a focused, high quality campaign (see Part I, section F).

The purpose of this document is to facilitate early detection and a rapid and appropriate public-health response to reduce morbidity and mortality due to measles in measles mortality reduction settings. It provides guidance on:

- definitions of cases and outbreaks;

- confirming, investigating and managing an outbreak, including appropriate vaccination strategies for outbreak response;

- $\quad$ learning lessons from the outbreak.

Information on measles disease, its differential diagnosis, transmission, immunity and the vaccine can be found in the WHO Module on best practices for measles surveillance (6), the WHO position paper on measles vaccine (7), and the WHO immunological basis for immunization measles module ( 8 ). 


\section{Goals of measles outbreak response}

The primary goal of measles outbreak response is to reduce morbidity and mortality by providing appropriate case management and vaccinating children.

Secondary goals are to:

- $\quad$ limit the spread of the outbreak;

- identify high-risk groups/areas for implementing strategies to improve vaccination coverage and other control measures;

- $\quad$ assist in the identification and correction of weaknesses in immunization and surveillance;

- $\quad$ raise awareness in the community about the disease and its prevention;

- monitor the changing epidemiology of measles.

\section{Target audience}

This document is targeted for use in countries with measles mortality reduction goals. It provides guidance to public-health workers at the national, regional or sub-national/ district levels on the appropriate epidemic prevention and outbreak response practices. The guidance provided in this document is not intended for countries with an elimination goal, or for emergency settings. In these latter settings, the goal is to stop transmission as soon as possible, and one confirmed case will require different recommendations for response - for guidelines in emergency settings see reference 9 , and see reference 10 for guidance on outbreak response in countries with an elimination goal $(9,10)$.

\section{E. Strategies for measles prevention and control: current recommendations}

In their Global Plan for Measles Mortality Reduction, 2006-2010 (11), WHO and UNICEF have targeted 47 priority countries with high measles burden. Most of the 47 priority countries are in Africa and South-East Asia, and they account for the overwhelming majority of global deaths from measles and its complications. This strategic plan includes the following key components:

a) Provide the first dose of measles vaccine to successive groups of all children at the age of nine months or shortly after.

b) Guarantee a 'second opportunity' for measles vaccination, either through campaigns or routine immunization. The second opportunity is needed both to increase the chance that every child receives at least one dose of measles vaccine, and to provide a second dose to children that already received one dose, thereby increasing the proportion of the population that is protected against measles. When the first dose is given at nine months, not all children will develop a protective response. The second dose, given later, will increase the protective response and the likelihood of immunity.

c) Establish an effective system to monitor coverage, and conduct measles surveillance with integration of epidemiological and laboratory information.

d) Improve clinical management of every measles case. 
Remarkable progress has been made in implementing the comprehensive strategy and reducing measles deaths in most of the 47 priority countries. Between 2000 and 2006, nearly 500 million children were vaccinated during measles supplementary immunization activities, either through catch-up or follow-up campaigns.

\section{F. Rationale for use of measles vaccine to control outbreaks}

As indicated in section A above, an extensive literature review of data (published from 1995-2006) to re-assess the impact of immunization response to measles outbreaks was carried out to provide the needed evidence to update the existing WHO recommendations on measles outbreak response published in 1999 (1). This literature review has challenged the existing WHO position which suggests that supplementary immunization activities in the setting of an outbreak undertaken with the aim of interrupting transmission of the virus may not have a substantial impact on the course of the measles outbreak.

More specifically, the recent articles reviewed show that:

1) outbreaks may last for months in a limited geographical area, and geographical spread of disease may be slow in areas with little population movement, thereby allowing sufficient time to mount an immunization response $(12,13)$;

2) outbreak response immunization was associated with reduced morbidity and spread of measles, particularly if it was started early, covered a wide age range, and achieved high coverage (14-21);

3) when planning vaccination response, it is important to consider measles control goals, background vaccination coverage, age distribution of cases, population density, population movement and case fatality rates. $(16,22)$.

The guidance provided in this document is intended to encourage the principles of early detection of measles outbreaks, thorough assessment, and a rapid response that includes expanded use of measles vaccine. The nature and extent of the vaccination response should be based on an assessment of risk of spread, risk of severe outcomes, and capacity to respond. Because of the diversity of situations in which outbreaks occur, a district outbreak coordination committee with broad representation should take the decisions as to the type of vaccination response at the local level. 


\section{Part II: \\ Definitions}

This section provides the definitions needed for outbreak confirmation and investigation.

\section{A. Case definitions for measles surveillance and outbreak investigation}

\section{Clinical case definition of suspected measles}

Any person in whom a clinician suspects measles

OR

Any person with:

- $\quad$ fever, and

- generalized maculopapular (i.e. non-vesicular) rash, and

- $\quad$ cough, coryza (i.e. runny nose) or conjunctivitis (i.e. red eyes).

\section{Laboratory criteria for diagnosis}

- $\quad$ Presence of measles-specific IgM antibodies is the recommended method for measles diagnosis ${ }^{1}$ (see Annex 1 for more details).

\section{Case classification}

- Clinically confirmed measles: Any person meeting the clinical case definition of suspected measles and cannot be discarded. ${ }^{2}$

- Laboratory-confirmed: A case that meets the clinical case definition and has laboratory-confirmation of measles virus infection.

- Epidemiologically confirmed: A case that meets the clinical case definition and is linked epidemiologically ${ }^{3}$ to a laboratory-confirmed or another epidemiologicallyconfirmed case.

1 Measles virus identification in a case that meets the clinical case definition of suspected measles is also a confirmation of a measles virus infection.

2 A suspected case in which laboratory investigations have equivocal results for measles-specific IgM antibodies after being tested twice, should be classified as a clinically confirmed measles case.

3 Epidemiological linkage: a case that meets the clinical case definition and has contact with a laboratory-confirmed measles case whose rash onset was within the preceding 21 days, or a case that lives in the same district or adjacent districts where a measles outbreak has been laboratoryconfirmed and transmission is plausible. 
- Discarded: A suspected case that has been subjected to serology or virus identification but has not met any of the laboratory criteria for diagnosis, or a suspected case which has been linked epidemiologically to a confirmed case of another communicable disease (e.g. rubella).

It is important to note that during an epidemic, most cases are clinically confirmed.

A measles-related death is a death in an individual with confirmed (clinically, laboratory, or epidemiologically) measles in which death occurs within 30 days of rash onset and is not due to other unrelated causes e.g. a trauma or chronic disease.

\section{B. Definition of a measles outbreak}

The definition of an outbreak will vary according to the phase of measles control. This guide recommends two different definitions for measles outbreaks depending on whether the country has conducted nationwide catch-up SIA's or not. These definitions are intended to provide general guidance only, and an assessment of the situation should be done on a case-by-case basis to determine if an outbreak is suspected or confirmed.

\section{For countries (or states/provinces of large countries) that have completed nationwide catch-up measles SIAs:}

A suspected outbreak of measles is defined as the occurrence of five or more reported suspected cases of measles in one month per 100000 population living in a geographical area (e.g. district/ block) (17).

A confirmed measles outbreak is defined as the occurrence of three or more confirmed measles cases (at least two of which should be laboratory-confirmed; IgM positive) in a health facility/district/block (approximate catchment population of 100000 ) in a month.

\section{For countries (or states/provinces of large countries) that have not yet completed nationwide catch-up SIAs:}

A suspected measles outbreak is defined as "an increase in the expected number of suspected measles cases being reported in a specific geographical area".

An increase in the number of suspected measles cases means a substantial increase in incidence compared to non-epidemic years, or incidence similar to the incidence in an epidemic year. For example, Table 1 shows the attack rates for the urban area of Niamey in the Republic of the Niger during the first calendar week of 2002-2004. In this example, it appears that 2004 was an epidemic year. However, when available, data for several weeks of the year and over at least five years should be taken into account to determine if a measles outbreak is suspected. 
Table 1: Attack rates per 100000 population in Niamey, Niger, first calendar week 2001 - 2004

\begin{tabular}{|c|c|c|}
\hline Year & Number of reported cases & $\begin{array}{c}\text { Attack rate } \\
\text { (per 100 000) }\end{array}$ \\
\hline 2002 & 2 & 0.3 \\
\hline 2003 & 1 & 0.1 \\
\hline 2004 & 60 & 7.2 \\
\hline
\end{tabular}

Source: Direction de la Surveillance et du Contrôle des Epidémies, Niger

A specific geographical area includes the catchment area of a health centre or a sector of a large city. In measles endemic regions, localized outbreaks occur within larger communities, with each local community experiencing outbreaks every 3-5 years. Hence, figures collected at regional and national level may not detect localized outbreaks.

If information from previous years is unavailable, the situation should be followed for the coming weeks. In this case, an ascending epidemic curve with an increasing number of cases over 3-4 weeks above the number seasonally expected should be considered as a suspected outbreak.

A confirmed measles outbreak is defined as the occurrence of three or more confirmed measles cases (at least two of which should be laboratory-confirmed; IgM positive) in a health facility/district/block (approximate catchment population of 100000 ) in a month.

If laboratory confirmation is not possible, an outbreak may be documented through a sustained and progressive rise in clinically-confirmed cases over a three-week period. In addition, hospitalization of a proportion of the suspected cases due to measles-related complications (e.g. diarrhea or respiratory infections such as pneumonia or croup), can be used to distinguish measles from outbreaks of other infections with fever and rash, where rates of hospitalization and related complications are usually much lower. 


\section{Part III: \\ Confirming, investigating and managing an outbreak}

The response to the outbreak needs to be developed fast and aggressively. The type of outbreak response varies, depending on a number of factors, including the level of susceptibility in the population, risk for spread and complications, and the existing health-service infrastructure.

In order to enhance the capacity to respond to measles outbreaks, a district level Outbreak Coordination Committee or any equivalent sub-national level multidisciplinary group should be created prior to the occurrence of outbreaks. The committee should be chaired by a government official, if available, and should include all potential partners (including NGOs working in the affected area) (see Annex 2). The role of the committee is to coordinate the outbreak risk assessment, and implementation and evaluation of the response.

The Outbreak Coordinating Committee should ensure that the following actions are carried out:

a) laboratory confirmation of the outbreak;

b) ensuring adequate clinical management of cases;

c) intensifying surveillance and notification of suspected cases;

d) assessing the risk of a large outbreak with high morbidity and mortality;

e) investigating a confirmed measles outbreak;

f) implementing control and preventive measures (including vaccination activities);

g) ensuring effective community involvement and public awareness.

It should be noted that the order of these actions (a to g above) is not intended to indicate a chronological order for their implementation. Many of these actions should be undertaken concurrently as soon as the outbreak is suspected or confirmed.

Figure 1 is a flow chart summarizing the actions that should be taken when a measles outbreak is suspected or confirmed. Next to each action item, there is a reference to the section of the document addressing the action item. 
Figure 1: A flow chart summarizing the actions that should be taken when a measles outbreak is suspected or confirmed

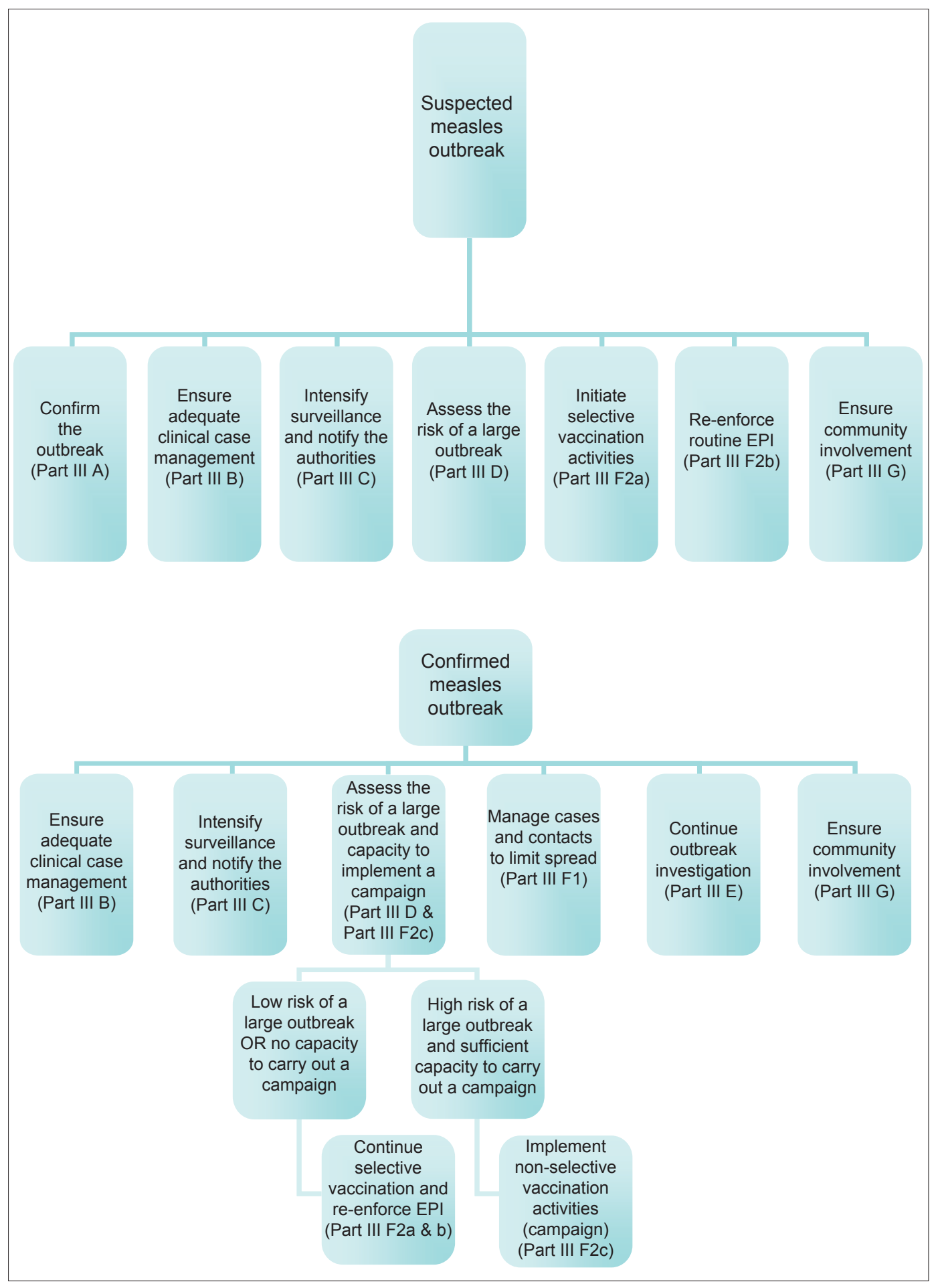




\section{A. Laboratory confirmation of a measles outbreak}

When an outbreak of measles is suspected it is critical to rapidly determine if the suspected outbreak is indeed a real outbreak by verifying that the reported suspected cases comply with the recommended clinical case definition, and that the increase in the number of reported cases meets the definition of an outbreak (see Part II). Whenever possible, all suspected measles outbreaks should be confirmed by the laboratory to be due to measles virus infection. In this, blood should be drawn from the first 5-10 suspected cases within an affected geographical area, for laboratory confirmation (the presence of measles-specific IgM antibodies) of the outbreak in that area. If there is suspicion that the outbreak has spread to an adjacent area, 5-10 blood specimens should also be collected from suspected cases in these areas, unless there is clear epidemiologic linkage between the two areas/districts. Once a measles outbreak is laboratory-confirmed, it is not necessary to collect specimens from every suspected case. Additional cases can be confirmed if they meet the clinical case definition and are epidemiologically linked to a laboratory-confirmed case.

For virus detection and sequence information, oropharyngeal swab specimens should be collected from five cases within the first seven days of rash onset from each outbreak. Sequence information can assist national control programmes, determine transmission pathways, and define geographical distribution of measles virus genotypes.

There is no need to incur delays while waiting for laboratory confirmation; response activities should be initiated as soon as there is a suspected outbreak (see definitions in Part II).

As mentioned in Part II above, if laboratory confirmation is not possible, an outbreak may be documented through a sustained and progressive rise in clinically-confirmed cases over a three- week period.

See Annex 1: Laboratory procedures

\section{B. Ensuring adequate clinical case management}

Significant morbidity and mortality are associated with measles. During an outbreak, adequate case management is critical in reducing measles mortality. There is currently no specific treatment for measles infection. Administration of vitamin A to children with measles has been shown to decrease both the severity of disease and the casefatality rate, and WHO recommends that vitamin A be administered to all children with acute measles. One dose (50 000 IU for infants aged less than six months, 100 000 IU for infants aged 6-11 months, and 200000 IU for children aged $\geq 12$ months) should be administered on the day of measles diagnosis, and one dose should be administered the following day. Supportive treatment should be provided for all cases, including additional fluids (such as oral rehydration solution) and antipyretics. Antibiotics should be used for cases complicated by otitis media or pneumonia, and nutritional therapy is indicated for children with malnutrition. Many children require four to eight weeks to fully recover their pre-measles nutritional status. 
According to the existing Integrated Management of Childhood Illnesses (IMCI) guidelines, prophylactic use of antibiotics is not recommended for uncomplicated cases. However, a recent study of measles cases in an impoverished community found prophylactic use of antibiotics was associated with improved outcomes (23). Detailed guidance on measles clinical case management is available in the WHO protocol for IMCI for treating measles complications (http://www.who. int/child-adolescent-health/integr.htm) and management of the child with a serious infection or severe malnutrition (24).

\section{Intensifying surveillance and notification}

\section{Intensify surveillance}

When an outbreak is suspected, surveillance should be intensified to ascertain the size and the geographical extent of the outbreak. As mentioned in Part II, whenever possible, all outbreaks should be laboratory-confirmed and, as already described, appropriate specimens should be taken from suspected cases.

Health staff at the health facility or district level should be vigilant and investigate any reports or rumors of measles cases occurring in the community, or when there is an epidemic occurring in a neighbouring area. Such investigations should either confirm (or reject) the existence of measles virus circulation.

At the health-facility level, measles surveillance should be intensified to actively seek additional cases. In this case, the following steps should be taken:

1) Investigate and collect data from each suspected case, adding the data to the linelisting form (see Annex 4). Data collected should at minimum include (name, address, age, sex, date of rash onset, vaccination status, date of last vaccination and, if applicable, date of specimen collection).

2) Institute weekly reporting, regardless of frequency of reporting prior to the outbreak.

3) If possible, conduct regular calls or visits to schools, hospital emergency rooms and other health facilities, plus selected paediatricians for case finding, especially in urban areas.

4) If time and resources permit, additional case finding should be conducted starting in health facilities where cases have already been reported, and including a search for cases in the community as follows:

a) search for additional cases in the registers;

b) look for patients who may have presented with similar signs and symptoms as measles;

c) request health staff to search for similar cases in registers of neighbouring health facilities;

d) ensure that private hospitals and clinics are included in the search;

e) conduct house-to-house visits in affected areas. 
Districts should aggregate data on measles cases and deaths (at minimum by age group, vaccination status and date of rash onset) and send weekly reports to the next (higher) administrative level, e.g. the Province (see also WHO Regional Office for Africa guidelines for measles surveillance (25).

\section{Notify the anthorities}

Once an outbreak has been laboratory-confirmed, block and district staff must immediately notify the next administrative level, e.g. the district (in case of block) or the Province (in the case of a district). The immediate notification report should include information on the number of cases and deaths by age group, vaccination status and date of rash onset, the geographical location of the outbreak and the activities planned to investigate and manage the outbreak. In addition, any supplies needed and technical support should be sought at this time (see section below).

Health authorities and health facilities at all levels and in nearby jurisdictions should be notified of the outbreak and updated as frequently as possible. This will allow them to be on the look-out for increases in the number of suspected measles cases and to begin appropriate preventive actions in their areas. If an importation from another country or Province may have occurred, the local health officials in the country or Province from which it was imported should be notified, and given all the information available. If a case has traveled or has had close contact with individuals from other areas of the country either during his/her exposure period (6-18 days before rash onset) or infectious period (i.e. from four days before to four days after rash onset), the surveillance coordinator in those areas should be notified immediately to alert them to the risk of measles outbreak in their areas.

Under the International Health Regulations (IHR) (2005), measles outbreaks could, under certain circumstances, be considered as Public Health Emergencies of International Concern (PHEIC). All measles outbreaks should be reported to the health authorities at the local, regional and national levels. At the national level, the decision instrument for the assessment and notification of events that may constitute PHEIC (outlined in Annex 2 of the IHR 2005, reference 5) should be used to determine if the measles outbreak should be notified to WHO. If so, the outbreak should be notified through the national IHR focal point.

\section{Assessing the risk of a large outbreak with high morbidity and mortality}

As soon as the outbreak is suspected, the risk of a large outbreak with high morbidity and mortality must be assessed. This assessment is needed to determine what type of vaccination response is most appropriate to control the outbreak. For this, the following evaluations should be carried out. 


\section{Evaluate the susceptibility of the population and potential for spread both in the affected and neighbouring areas.}

Approximately $15 \%$ of children vaccinated at nine months of age and $5 \%-10 \%$ of those vaccinated at 12 months of age fail to seroconvert, and are thus not protected after vaccination. The example of district $\mathrm{X}$ with a population of 500000 and 12500 births per year illustrates the build up in susceptibles. If $80 \%$ of children aged nine months receive measles vaccination through routine health services, and assuming $85 \%$ vaccine effectiveness, only 8500 children $(12500 \times 0.8 \times 0.85$, or $68 \%$ ) in each birth cohort of 12500 will be protected against measles, and 4000 children $(32 \%)$ will remain susceptible to measles. Thus, 4000 children will be added each year to the pool of measles-susceptible children. As a general guide, an outbreak is likely to occur when the pool of susceptible children reaches the size of one birth cohort. In this example, an outbreak is likely to occur in district $\mathrm{X}$ after 3-4 years (see Table 2). It should be noted that this method only provides an approximation of the susceptibles in a population (i.e., those arising from new births) as it does not take into account other sources of susceptibles in the population. For example, the susceptibles in the population in the age beyond the target age group, or susceptibles migrating into the population are not included.

Table 2: Table showing an approximation of the buildup of susceptible children with each successive birth cohort over a four-year period in the example of district $\mathrm{X}$ above

\begin{tabular}{|c|c|c|c|}
\hline Year & $\begin{array}{c}\text { Cumulative No. } \\
\text { of live births }\end{array}$ & $\begin{array}{c}\text { Cumulative No. } \\
\text { of children protected } \\
\text { against measles }\end{array}$ & $\begin{array}{c}\text { Cumulative No. } \\
\text { of children susceptible } \\
\text { to measles }\end{array}$ \\
\hline 1 & 12500 & 8500 & 4000 \\
\hline 2 & 25000 & 17000 & 8000 \\
\hline 3 & 37500 & 25500 & 12000 \\
\hline 4 & 50000 & 34000 & 16000 \\
\hline
\end{tabular}

To estimate the susceptibility profile by age group, the proportion of susceptible persons should be calculated by taking into account the estimated population and the vaccination coverage of each age group from six months to 14 years and 11 months of age (through both routine immunization and campaigns) and estimated vaccine efficacy. 
2. Evaluate the risk of further transmission, morbidity and mortality. For this, the following factors should be taken into account.

- $\quad$ Population characteristics such as size, density, movement, and setting (e.g. community spread throughout a district, or limited spread within a subpopulation; resource poor settings).

- Under five mortality rates.

- Nutritional, including vitamin A, status.

- HIV prevalence in the population.

- $\quad$ Period of the year (considering potential for seasonal outbreak) and plans for any festivals or other social events that will result in increased opportunities for spread.

- Number of cases reported and comparison with data from previous years.

- Access to health services.

\section{E. Investigating a confirmed measles outbreak}

\section{Collect data}

During an outbreak, data collection should be limited to obtaining basic information from each case (name, address, age, sex, immunization status, date of last vaccination, symptoms, date of rash onset, date of specimen collection, outcome), which should then be added to the line-listing. Any additional information on the population where the outbreak occurs that may shed light on the presence of risk factors for measles infection should, if possible, be obtained (e.g. available information on the HIV prevalence or nutritional status of the population). The data should be analysed rapidly to determine the extent and severity of the outbreak, vaccine effectiveness, potential risk factors for measles infection, and possible causes of the outbreak (e.g. vaccine failure or failure to vaccinate).

\section{Describe the outbreak}

Describe the outbreak in terms of time, person, and place.

- Time: What are the dates of rash onset among cases? Allows the creation of the epidemic curve.

- Person: What are the characteristics of the cases (e.g. age distribution, vaccination status)?

- Place: Where do cases live? Where are the most affected areas/localities? Allows the mapping of the geographical extent of the outbreak (e.g. spot map or table with attack rates by district).

Details and examples of methods defining the extent of the outbreak can be found in the WHO Module on best practices for measles surveillance (6). 


\section{Analyse data}

Data collected should be analysed by district-level staff to gain a better understanding of the outbreak and use the information to guide the outbreak response activities. Below are important indicators that provide information on the severity of the outbreak. For information on how these are calculated see Annex 3.

\section{Attack rate (AR)}

The AR expresses the number of cases among the total population in a given area during a defined period of the outbreak. If population data by age group are available for the area affected by the outbreak, age-specific attack rates can be calculated. Age-specific attack rates help to identify the age ranges for priority vaccination. AR allow for comparison of the extent of the outbreak between different populations (e.g. by age group or by geographical location). For example, Table 3 shows the attack rates by commune (districts within the city) in Niamey in the Republic of the Niger for the epidemic in 2003-2004.

Table 3: Attack rates for measles by commune of origin of patients, Niamey, Niger (1 November 2003 - 6 June 2004)

\begin{tabular}{|c|c|c|c|}
\hline Commune & Population & No. cases & Attack rate (\%) \\
\hline I & 347567 & 5789 & 1.7 \\
\hline II & 306772 & 3598 & 1.2 \\
\hline III & 115115 & 587 & 0.5 \\
\hline Total & 769454 & $9974^{*}$ & 1.3 \\
\hline
\end{tabular}

* For 906 cases, information about the commune of origin was missing.

Source: Direction de la Surveillance et du Contrôle des Epidémies, Niger

It should be emphasized that it is important to determine the place where the suspected case was infected rather than the place from where he/she may seek care, as patients may seek health care in a different area to where they live. This information helps to follow the geographical spread of the epidemic and identify areas at higher risk. Ideally, it is also important to determine the attack rate by age group.

\section{Weekly incidence}

Weekly incidence is the number of new cases of the disease by week in a specified population. Attack rates and weekly incidence numbers permit comparison between different geographical areas and monitor the progression of the outbreak over time. 


\section{Case-fatality ratio (CFR)}

The case-fatality ratio measures the proportion of deaths ${ }^{4}$ among confirmed measles cases. It should be calculated for the community as a whole as well as hospitals. In the community, the CFR may be underestimated as many cases may die at home and where deaths are not reported. A high CFR in hospitals may be interpreted in many different ways. For example, that case management is not effective, or that mainly severe cases or those in extremis seek hospital care. If a more accurate estimate of the CFR is required, a carefully conducted community-based study should be undertaken. The formula for calculating CFR can be found in Annex 3, and where possible CFR should be estimated by different age groups.

\section{Vaccine effectiveness (VE)}

VE measures the effectiveness of vaccination in conferring protection against measles. The information required and formula for calculating VE is outlined in Annex 3.

For a more detailed analysis, a case-control study is needed to elucidate more specific risk factors, such as age at vaccination, number of doses received, access to care and travel history. Cases and controls (persons without measles) are identified and then compared to determine to what extent they differ, e.g. by age, sex, vaccination status, etc. To obtain assistance for such a study, district officials should consult with epidemiologists at the provincial and national level.

\section{Interpret data}

Generally, the initial descriptive analysis of person, time and place will help define who is at risk of measles and which areas are affected. In addition, data on the age of cases and their vaccination status will help to identify causes of the outbreak and the population that is at highest risk. This information is needed to guide the response activities, for example, defining the population and the age groups to be targeted for vaccination. When interpreting data, it is important to take into consideration the quality of the surveillance system generating the data, as reliable information is needed to guide action.

4 A measles-related death is a death in an individual with a confirmed (clinically, laboratoryconfirmed or epidemiologically) case of measles in which death occurs within 30 days of rash onset and is not due to another unrelated cause e.g. a trauma or chronic disease. 


\section{F. Implementing control and preventive measures}

\section{Managing cases and contacts to limit spread}

As discussed in section $\mathrm{B}$ above, it is important to ensure adequate clinical management of measles cases in order to reduce measles mortality. In addition, if time and resources permit, the following measures should be implemented, as follow-up of all cases and contacts may not be possible if the epidemic is large and if resources are limited.

Health staff should report all patients in whom they suspect measles. Monitoring and follow-up of suspected cases includes the following measures.

- Limiting contact to only immediate family members who have been vaccinated or have prior history of measles. In particular, avoid contact with infants or young unimmunized children in the household.

- $\quad$ Suspected cases should not be hospitalized, unless they have complications or another condition requiring hospitalization, because of the high risk of intra-hospital transmission.

- $\quad$ Patients with measles who require hospitalization should, if possible, be isolated from onset of prodromal symptoms until five days after rash onset; health staff in contact with these patients should use respiratory precautions during this period

- Contact should be limited in outpatient departments (OPDs) such as waiting rooms, where there are suspected cases. For example, where feasible, separate segregated waiting areas and examination rooms for suspected cases should be set up in outpatient clinics.

Also, where feasible, officials must be prepared to identify persons who have had contact with a confirmed measles case and take the following actions to minimize spread.

- Contacts without written evidence of measles vaccination should be vaccinated and the symptoms of measles should be explained to them.

- $\quad$ During the second week after exposure, and at the first sign of possible measles (fever, runny nose, cough or red eyes) the contact should be instructed to stay at home.

\section{Conducting appropriate vaccination activities}

The district level Outbreak Coordinating Committee responsible for outbreak response should determine the appropriate vaccination activities following the steps outlined in Annex 2.

As soon as an outbreak is suspected, steps (a) and (b) below should be undertaken concurrently. In addition, as soon as the outbreak is confirmed, the district Outbreak Coordination Committee should review risk-assessment results and decide accordingly whether to continue with the selective vaccination activities or to carry out a nonselective vaccination campaign (see Figure 1). 
a) Selective vaccination activities.

As soon as a measles outbreak is suspected, the following steps should be taken.

i) Enhance social mobilization activities to inform the affected communities about the suspected outbreak, which specific age group of previously unvaccinated children is targeted for measles vaccination, and where parents should bring their at-risk children for vaccination.

ii) Vaccinate all children (six to 59 months of age or determine the target age group according to the local disease epidemiology) presenting to a health facility or an outreach vaccination site without a history of measles vaccination (either written or verbal). Children receiving measles vaccine before the age of nine months must be revaccinated after the age of nine months (with at least a one-month interval between the doses). Note that in these mortality reduction settings the majority of measles cases occur in children under 5 years of age $(21,26)$.

iii) Vaccinate hospital staff at risk of exposure who have not been vaccinated.

iv) Ensure sufficient supplies. Use stock management records to determine available quantity and location of vaccine, auto-disable (AD) syringes and other supplies (e.g. cold-chain equipment and vitamin A) that are available immediately for use. Estimate and request the additional supplies needed so that activities are not interrupted due to supply stock outs.

b) Reinforcement of routine vaccination.

A measles outbreak provides the opportunity to identify programme weaknesses causing the outbreak and a chance to correct them. As soon as a measles outbreak is suspected, and before laboratory confirmation of the suspected measles cases, the following steps should be taken to reinforce routine vaccination.

i) District staff, health-facility staff and partners should rapidly identify priority areas within the affected district (e.g. communities with low vaccination coverage and at high risk of morbidity and mortality).

ii) Jointly work on strengthening the available district immunization workplans.

iii) Locate health centres conducting immunization sessions that may need additional staff or vaccine supplies.

iv) Organize corrective measures such as additional outreach services to communities with a high proportion of unreached children.

c) Non-selective mass vaccination activity.

As soon as the outbreak is confirmed, and if the risk-assessment results indicate that there is a high risk of a large measles outbreak, then the capacity to carry out a high quality large-scale immunization campaign should be rapidly evaluated. That is:

- evaluate the availability of staff and financial resources (both internal and external) for the operational and logistical aspects of the campaign;

- $\quad$ evaluate if the vaccine and other supplies can be made available within the timescale necessary. 
Carry out a safe and timely vaccination campaign in the targeted areas (affected and neighbouring areas as determined by the risk assessment) as soon as there is sufficient capacity (human and financial resources and vaccine and other supplies).

However, if the outcome of the risk assessment does not indicate a mass vaccination response, then selective immunization of unimmunized children presenting to health facilities as outlined in step (a) above should be continued, and the number of reported cases closely followed to monitor the progression of the outbreak.

For the non-selective mass vaccination response, the timing, target age group and area for vaccination, should be defined as outlined below. An accelerated micro-planning exercise should be performed to determine the bundled vaccine, logistics, staffing and communications needs for the campaign. Existing country or regional guidelines for conducting mass measles vaccination campaigns should be used (see the WHO Regional Office for Africa: measles SIAs field guide (27).

Timing of intervention: Once the decision to intervene has been made, it is critical to act as quickly as possible to minimize the number of severe measles cases and deaths.

The timing of the intervention plays a key role in the number of cases and deaths that may potentially be prevented. For example, Figure 2 shows the epidemic curve for N'djamena in the Republic of Chad in 2004-2005. An intervention was proposed at the beginning of the epidemic, and in the middle, and was finally implemented about five months after the start of the epidemic. Had the intervention occurred earlier, it is clear that its impact would have been greater. Even though it occurred late in the epidemic, the intervention may have contributed to improving population immunity, shortening the duration of the outbreak and preventing some severe cases and deaths.

Figure 2: The epidemic curve for N'djamena, Chad in 2004-2005

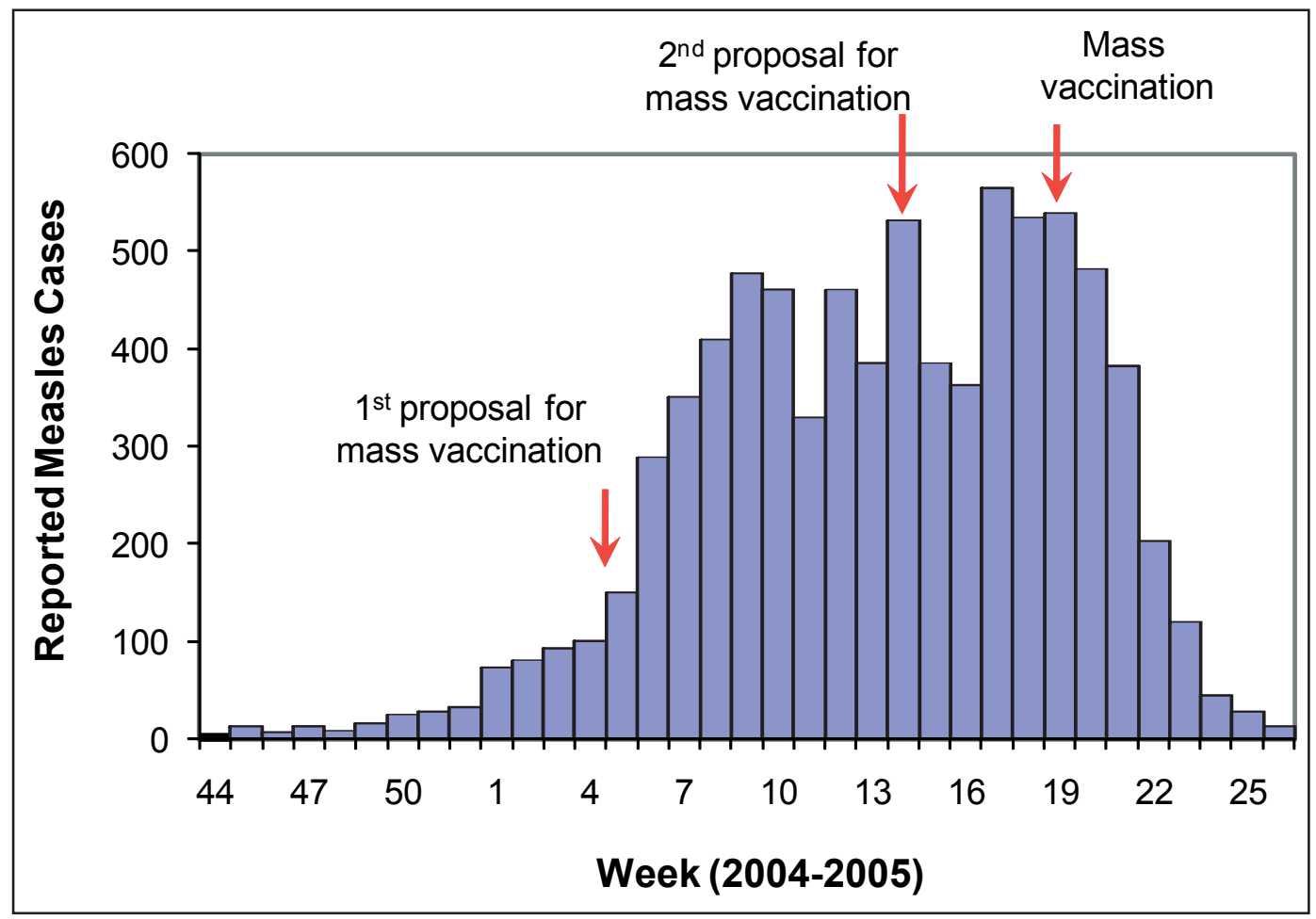




\section{Target population}

Choosing the target population depends upon the susceptibility profile of the population. Key elements to consider are:

1) routine vaccination coverage and coverage during SIAs in each birth cohort;

2) age-specific attack rates;

3) absolute number of cases.

For example, Table 4 shows the attack rates and population and number of cases during an epidemic in Niamey, the Republic of the Niger, in 1995. The highest attack rate occurs in the 6-8 month age group. Although the attack rate in the 5-14 year old age group is much lower, the absolute number of cases in this age group is almost $25 \%$. It is important to consider both absolute numbers of cases and the potential at-risk population, as well as the attack rate. In this example, the 5-14 year old age group may also contribute to transmitting the disease to younger age groups and so could be targeted for vaccination.

Table 4: Measles attack rates (AR) by age groups in Niamey, Niger, 1 January - 7 May 1995

\begin{tabular}{|l|c|c|c|}
\hline \multicolumn{1}{|c|}{ Age groups } & Population & Cases & AR\% \\
\hline $0-5$ months & 12777 & 408 & 3.2 \\
\hline $6-8$ months & 5555 & 1139 & 20.5 \\
\hline $9-11$ months & 6667 & 878 & 13.2 \\
\hline $12-59$ months & 82772 & 6908 & 8.3 \\
\hline $5-14$ years & 155544 & 3607 & 2.3 \\
\hline$\geq 15$ years & 292201 & 935 & 0.3 \\
\hline Total & $\mathbf{5 5 5 5 1 6}$ & $\mathbf{1 3 8 7 5}$ & $\mathbf{2 . 5}$ \\
\hline
\end{tabular}

It is critical that the results from the outbreak investigation and prior surveillance data be used to develop and tailor an appropriate and logical response, e.g. to determine additional age and risk groups to be targeted for vaccination. If, for example, the data suggest that older children are affected, then the age group initially targeted for vaccination should be adjusted to include older cohorts. All age groups contributing to cases should be considered for vaccination. Even if the attack rate is low in some age groups, especially in older groups, they may represent a large proportion of cases and large potential groups at risk of both contracting measles and subsequent complications, or of transmitting the infection to younger persons. Once the age group targeted for vaccination is determined, all children in that age group should be vaccinated regardless of their vaccination status. 


\section{Target area}

The response should target both outbreak-affected areas and adjacent areas in which the risk- assessment shows a high risk of spread. As distinct from preventive SIAs (e.g. catch-up and follow-up campaigns) that target whole countries, provinces or states, campaigns done in response to outbreaks should be more limited in scale.

Health staff should pay particular attention to ensure that groups and areas with a high likelihood of not being reached (i.e., with known low coverage) and at high risk for measles-related complications are reached during the vaccination activities, and any necessary supplemental measures such as the provision of vitamin A are provided. These vulnerable groups and areas include:

- $\quad$ young children, particularly those under one year of age;

- malnourished and vitamin A-deficient children;

- $\quad$ infants and children of HIV-infected women, and other immunocompromised children;

- certain ethnic and religious groups who may have poor access to immunization;

- $\quad$ populations with poor access to health care;

- $\quad$ staff at hospitals and other health facilities;

- All children above six months of age who are attending hospitals (inpatients and outpatients) or who are visiting the hospital.

Children receiving measles vaccine before the age of nine months during a campaign must be revaccinated after the age of nine months (with at least a one-month interval between the doses), since the efficacy of vaccine administered before nine months of age is likely to be low. Strategies to ensure that these children receive a second dose of measles vaccine include the following steps:

- $\quad$ informing mothers at the time of vaccination that their child must be vaccinated again;

- $\quad$ notifying health workers, NGOs and the community, about the need for these infants to receive a second dose.

\section{Target Coverage}

Ideally, the target coverage (the proportion of the target population you want to vaccinate) should be $100 \%$. However, although this may not always be possible given available resources, the intervention should be conducted, even if it is not possible to achieve $100 \%$ coverage. Once the vaccination activities are conducted, it is important to carry out rapid coverage monitoring to estimate the coverage achieved, and to identify potential groups of missed children and ensure that they are vaccinated. 


\section{G. Ensuring effective community involvement and public awareness}

When an outbreak is confirmed, there is likely to be widespread public concern and media attention. It is important to keep the public informed, to calm fear and encourage cooperation. Messages to the community should be clear and concise, using local terminology, and should convey the following:

- $\quad$ existence of an outbreak and the benefits of measles vaccination;

- $\quad$ signs and symptoms of the disease;

- $\quad$ encouragement to parents whose children have had a recent rash and fever illness to consult a health-care facility early after symptom onset;

- instruction to parents to bring their children to a health facility/vaccine post for vaccination;

- information on locations and opening hours of health facility/vaccine posts.

Messages to the community can be transmitted by radio and/or television, newspapers, posters and fliers, meetings with health personnel, community, religious and political leaders, and presentations at markets, health centres and schools. The media are useful partners in keeping the public informed through regular press releases and conferences. Select and use a community spokesperson to serve as focal person for the media. As soon as the outbreak has been recognized:

- tell the media the name of the spokesperson, and that all information about the outbreak will be provided by the spokesperson;

- $\quad$ release information to the media only through the spokesperson to make sure that the community receives clear and consistent information.

Meet with the spokesperson on a regular basis to give:

- frequent, up-to-date information on the outbreak and response;

- $\quad$ clear and simple health messages for the media to use. 


\section{Part IV: \\ Learning lessons from the outbreak}

After the outbreak, the outbreak coordination committee should perform an evaluation of:

- Measles surveillance and the timeliness of outbreak detection.

- The cause of the epidemic. For example, if the outbreak is caused by failure to vaccinate children or certain groups, then the outbreak coordination committee should investigate the reasons for failing to reach these groups through the existing Expanded Programme on Immunization (EPI). Alternatively, if the reason for the outbreak is determined to be vaccine failure, then steps should be taken to investigate the causes of the vaccine failure (e.g. failure of the cold chain in the outbreak area).

- Preparedness for the epidemic.

- Management of the epidemic, including an evaluation of the curative and vaccination intervention.

- Evaluation of costs, including impact on other health-delivery programmes.

- The goals and operations of the immunization programme.

- The implications for developing appropriate response strategies for future outbreaks.

Feedback is critical, and the findings should be indicated in a written report containing clear description of the epidemiological characteristics of the epidemic and recommendations on:

- immunization strategies for addressing weaknesses in EPI, to increase coverage and target high-risk areas in order to prevent future outbreaks;

- $\quad$ improving surveillance;

- $\quad$ preparedness and the improvement of response to outbreaks. 


\section{Annex 1: Laboratory procedures}

For further details, see the measles laboratory manual ${ }^{5}$

\section{Collection and shipment of single blood specimens for IgM serology}

Blood specimens should be collected at the first contact the person has with the healthcare provider, but within 28 days of rash onset. Collection procedure is as follows:

- $\quad$ Collect $5 \mathrm{ml}$ blood by venepuncture into a duly-labeled sterile tube.

- Whole blood should be centrifuged at $1000 \mathrm{xg}$ for 10 minutes to separate the serum.

- Blood can be stored at $4-8{ }^{\circ} \mathrm{C}$ for up to 24 hours before the serum is separated.

- $\quad$ Do not freeze whole blood.

- If there is no centrifuge, blood should be kept at room temperature $\left(4-30{ }^{\circ} \mathrm{C}\right)$ until there is complete retraction of the clot from the serum.

- Carefully remove the serum, avoiding extracting red cells, and transfer aseptically to a sterile labeled vial.

- Store serum at $4-8{ }^{\circ} \mathrm{C}$ until it is ready for shipment. The serum can be stored in refrigeration for a maximum period of seven days. Serum must be frozen at $-20{ }^{\circ} \mathrm{C}$ if it is going to be stored for longer periods.

A second specimen may occasionally be required if the first specimen had an equivocal result, or if the clinician needs to consolidate the diagnosis on an individual patient who gave an initial negative result. The second specimen may be collected at any time between 3-28 days after rash onset, preferably at least 10 days after the first specimen.

Virus isolation should be performed to identify the measles genotype causing the outbreak. Urine or nasopharyngeal samples need to be collected from 5-10 cases and sent to specialized reference laboratories - for more details see the AFRO Guidelines for Measles Surveillance (25).

5 http://www.who.int/immunization_monitoring/laboratory_measles_resources/en/index. html 


\section{Storage of specimens outside the laboratory}

Whole blood may be held at refrigerator temperature $\left(4-8^{\circ} \mathrm{C}\right)$ if it can be transported to arrive at the testing laboratory within 24 hours. If this is not possible, the tube must be centrifuged to separate the serum, which is transferred to a sterile, labeled screw-capped tube for transport to the laboratory. Sterile serum must be shipped on wet ice within 48 hours, or stored at $4-8^{\circ} \mathrm{C}$ for a maximum period of seven days.

For longer periods, sera must be frozen at $-20^{\circ} \mathrm{C}$ and transported to the laboratory on frozen icepacks. Repeated freezing and thawing can have detrimental effects on the stability of IgM antibodies.

\section{Shipment of specimens}

Specimens should be shipped without delay, and should be accompanied by a specimen form indicating date of collection and type of specimen, date sent to the laboratory, date of arrival, and date of results. A laboratory request form should also be completed at the time of specimen collection and should accompany all shipments. The latter form should include the following data: patient identification (unique ID, name, place of residence, age); basic clinical information (date of onset of rash, whether patient fulfils case definition), and immunization history (number of doses of measles-containing vaccine, date of last dose). Shipment procedures are as follows:

- $\quad$ place specimens in zip lock or plastic bags;

- $\quad$ use Styrofoam boxes or a thermos flask;

- $\quad$ place specimen form in plastic bag and tape to inner top of Styrofoam box;

- $\quad$ if using ice packs (these should be frozen), place ice packs at the bottom of the box and along the sides, place samples in the centre, then place more ice packs on top;

- $\quad$ arrange shipping date;

- when arrangements are finalized, inform receiver of time and manner of transport. 


\section{Annex 2: \\ The outbreak coordination committee}

A measles outbreak coordination committee at the district level should be formed prior to the occurrence of the measles outbreak. The committee should meet periodically to coordinate epidemic preparedness and to plan and review response activities. The outbreak coordination committee may include representatives from:

- $\quad$ the district medical office;

- $\quad$ the Ministry of Health

- $\quad$ district or provincial EPI manager;

- disease control officers for communicable diseases;

- national outbreak response officer;

- $\quad$ officer in charge of drug supply;

- $\quad$ hospitals (clinicians and nurses) and laboratories;

- NGOs e.g. Red Cross and managers of outreach programmes to special populations;

- $\quad$ community health programmes;

- $\quad$ police and other public safety officers;

- community leaders and representatives;

- $\quad$ private-sector representatives (e.g. officials from a private hospital, clinic or laboratory).

\section{The responsibilities of the outbreak coordination committee}

- $\quad$ meet in the absence of an outbreak to plan for outbreaks;

- $\quad$ assess the supplies and equipment and resources currently available;

- $\quad$ estimate and identify resources and procedures for preventive mass vaccination campaigns;

- $\quad$ estimate and identify additional resources needed for rapid outbreak response;

- $\quad$ ensure the availability of staff and training for outbreak response;

- $\quad$ analyse epidemiological information as the outbreak progresses;

- $\quad$ assign responsibilities to staff with clear tasks and lines of communication;

- meet regularly to review data and monitor implemented measures;

- communicate with the general public and the media;

- $\quad$ evaluate the response;

- $\quad$ evaluate the immunization programme;

- $\quad$ produce a detailed report on outbreak response activities;

- make recommendations on any changes to vaccination strategies and programmes. 


\section{Roles and responsibilities at different administrative levels}

The responsibilities of health authorities at district, regional, and national level during an outbreak must be clear. The district/regional/provincial level coordinates individual health facilities and plans and coordinates overall outbreak control activities. The national level coordinates outbreak control measures within the entire country, and international help, if appropriate, along with WHO and other partners. The appropriate international and nongovernmental organizations (NGOs) should become involved as early as possible. The following table outlines the responsibilities at each level. 


\begin{tabular}{|c|c|c|}
\hline Local health facility & District/regional/provincial level & National level \\
\hline $\begin{array}{l}\text { 1. Surveillance and } \\
\text { reporting: } \\
\text { - recognize cases of } \\
\text { measles; } \\
\text { - collect information on } \\
\text { cases and contacts; } \\
\text { arrange for laboratory } \\
\text { confirmation of first } \\
\text { 5-10 cases. } \\
\text { 2. Management of cases: } \\
\text { - confirm cases; } \\
\text { - provide treatment; } \\
\text { 3. Supply and logistics: } \\
\text { - determine needs and } \\
\text { request additional } \\
\text { support as needed. } \\
\text { 4. Public information } \\
\text { management and public } \\
\text { education: } \\
\text { - provide information } \\
\text { on the outbreak and } \\
\text { control activities to } \\
\text { the proper higher } \\
\text { authorities; } \\
\text { promote public } \\
\text { awareness of the } \\
\text { outbreak and what } \\
\text { the community } \\
\text { should do. }\end{array}$ & $\begin{array}{l}\text { 1. Surveillance and review of } \\
\text { data. } \\
\text { 2. Investigation of suspected } \\
\text { outbreaks. } \\
\text { 3. Intervention planning: } \\
\text { - define the vaccination } \\
\text { strategy; } \\
\text { define the geographical area } \\
\text { to be covered; } \\
\text { define the target age group } \\
\text { to be vaccinated and } \\
\text { estimate the total number of } \\
\text { individuals in the target age } \\
\text { group; } \\
\text { define time frame for } \\
\text { vaccination intervention; } \\
\text { define resources needed } \\
\text { including } \\
\text { - vaccines and injection } \\
\text { material; } \\
\text { - human resources; } \\
\text { outbreak surveillance data to } \\
\text { relevant government authorities } \\
\text { and health facilities. } \\
\text { Commence planning for an } \\
\text { evaluation of the intervention. } \\
\text { - cold chain and logistic } \\
\text { support including } \\
\text { transport; } \\
\text { - communication } \\
\text { information; } \\
\text { define a budget and potential } \\
\text { sources of funding for the } \\
\text { intervention; } \\
\text { ensure that there is ongoing } \\
\text { evaluation of the intervention } \\
\text { by measuring vaccination } \\
\text { coverage to verify that the } \\
\text { objective is being achieved. } \\
\text { strategy needed to increase } \\
\text { involvement, monitoring the } \\
\text { - }\end{array}$ & $\begin{array}{l}\text { 1. Public information } \\
\text { management: } \\
\text { notifying the outbreak } \\
\text { to WHO; } \\
\text { - organizing laboratory } \\
\text { confirmation } \\
\text { and shipment of } \\
\text { specimens for viral } \\
\text { isolation; } \\
\text { - convening a national } \\
\text { outbreak coordinating } \\
\text { committee and } \\
\text { assigning duties; } \\
\text { assisting in field } \\
\text { investigation; } \\
\text { providing assistance in } \\
\text { obtaining emergency } \\
\text { supplies, technical and } \\
\text { support personnel; } \\
\text { assessing and } \\
\text { obtaining resources. }\end{array}$ \\
\hline
\end{tabular}




\section{Annex 3: Formulae}

\section{Attack Rate (AR)}

If population data are available for the area of the outbreak, age-specific attack rates can be calculated, for instance:

$$
\mathrm{AR}_{0 \text { to } 11 \text { months }}=\frac{\text { number of cases in children aged } 0 \text { to } 11 \text { months }}{\text { total number of children aged } 0 \text { to } 11 \text { months }}
$$

Attack rates should also be calculated for other age groups. The denominator can be further refined to include only the population at risk, i.e. excluding those already vaccinated or who have suffered measles previously. This may give a more accurate attack rate among the susceptible population. The population included in the denominator and the period of time during which cases occurred should always be clearly stated.

\section{Case-fatality ratio (CFR)}

The CFR is an indicator of the severity of the outbreak. The CFR can be calculated as follows:

$$
\text { CFR }=\frac{\text { number of cases who died of measles }}{\text { total number of cases of measles }}
$$

For more information on CFR estimation, see reference 28.

\section{Vaccine effectiveness (VE)}

VE can be estimated from the data collected during an outbreak investigation and from routine coverage data. The coverage must be known or estimated for the age group in which cases are occurring.

This method is based on the difference between the attack rates among vaccinated persons (ARV) and those among the unvaccinated (ARU), expressed as a fraction of the attack rate among unvaccinated persons (ARU) - the greater the proportional reduction of illness in the vaccinated group, the greater the vaccine effectiveness.

$$
\mathrm{VE}=(\mathrm{ARU}-\mathrm{ARV}) / \mathrm{ARU}
$$


Vaccine effectiveness can also be estimated by plotting the percentages of measles cases in vaccinated individuals (PCV) and the percentage of the population vaccinated (PPV \%) on a nomogram, which shows the relationship between PPV, PCV and VE (see Figure 3). For instance, if $20 \%$ of measles cases are from individuals vaccinated against measles, and if vaccination coverage is $80 \%$, vaccine effectiveness is close to $95 \%$. If $60 \%$ of measles cases are from individuals vaccinated against measles, and if vaccination coverage is $80 \%$, vaccine effectiveness is close to $70 \%$.

More accurate determinations of vaccine effectiveness require intensive cohort or case-control studies and are beyond the scope of this document.

Figure 3: The relationship between percentage of cases vaccinated (PCV) and percentage of the population vaccinated (PPV)

for seven different percentage values of vaccine effectiveness (VE)

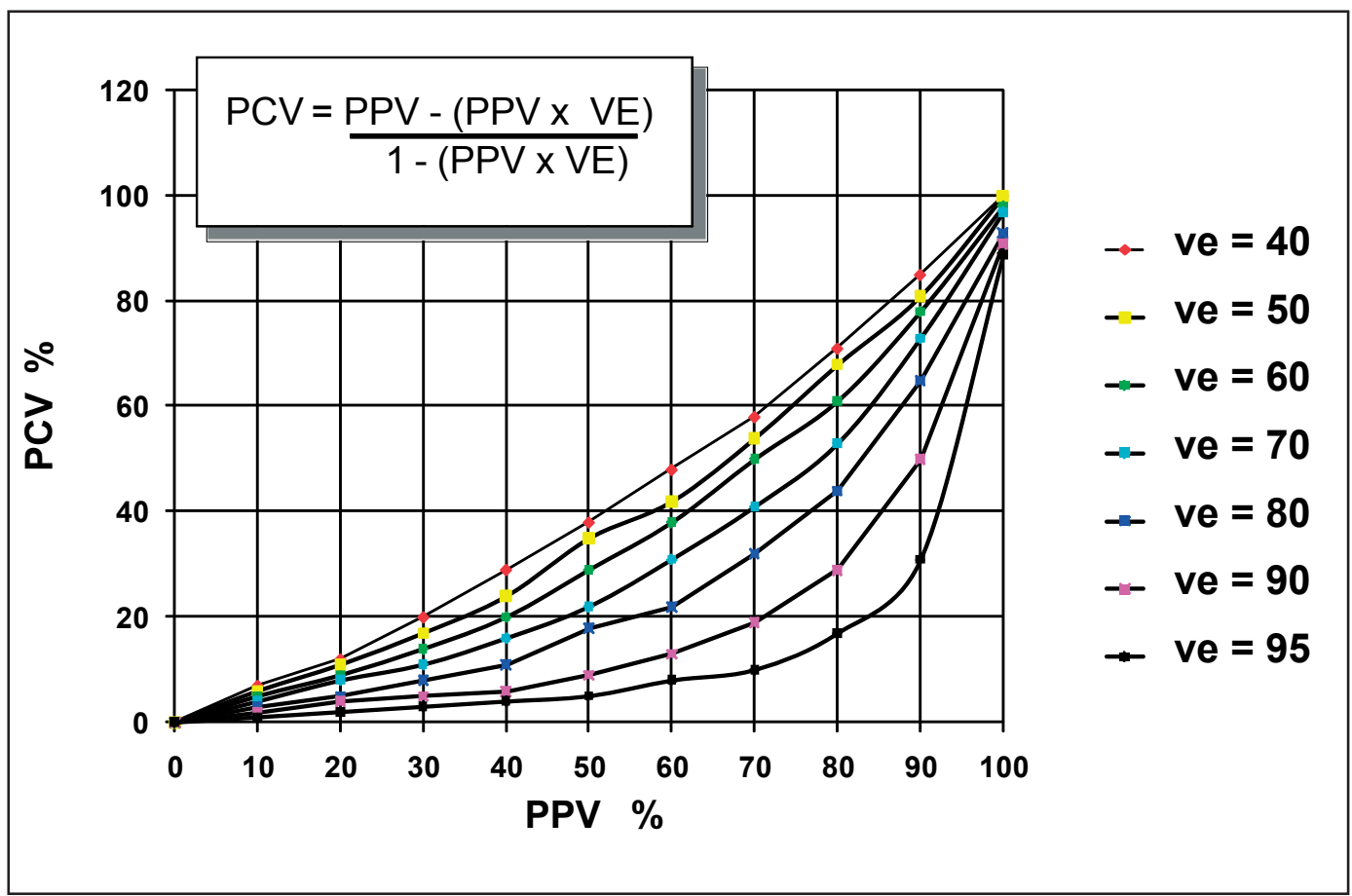




\section{Annex 4: \\ Generic line-list for use during measles outbreaks}



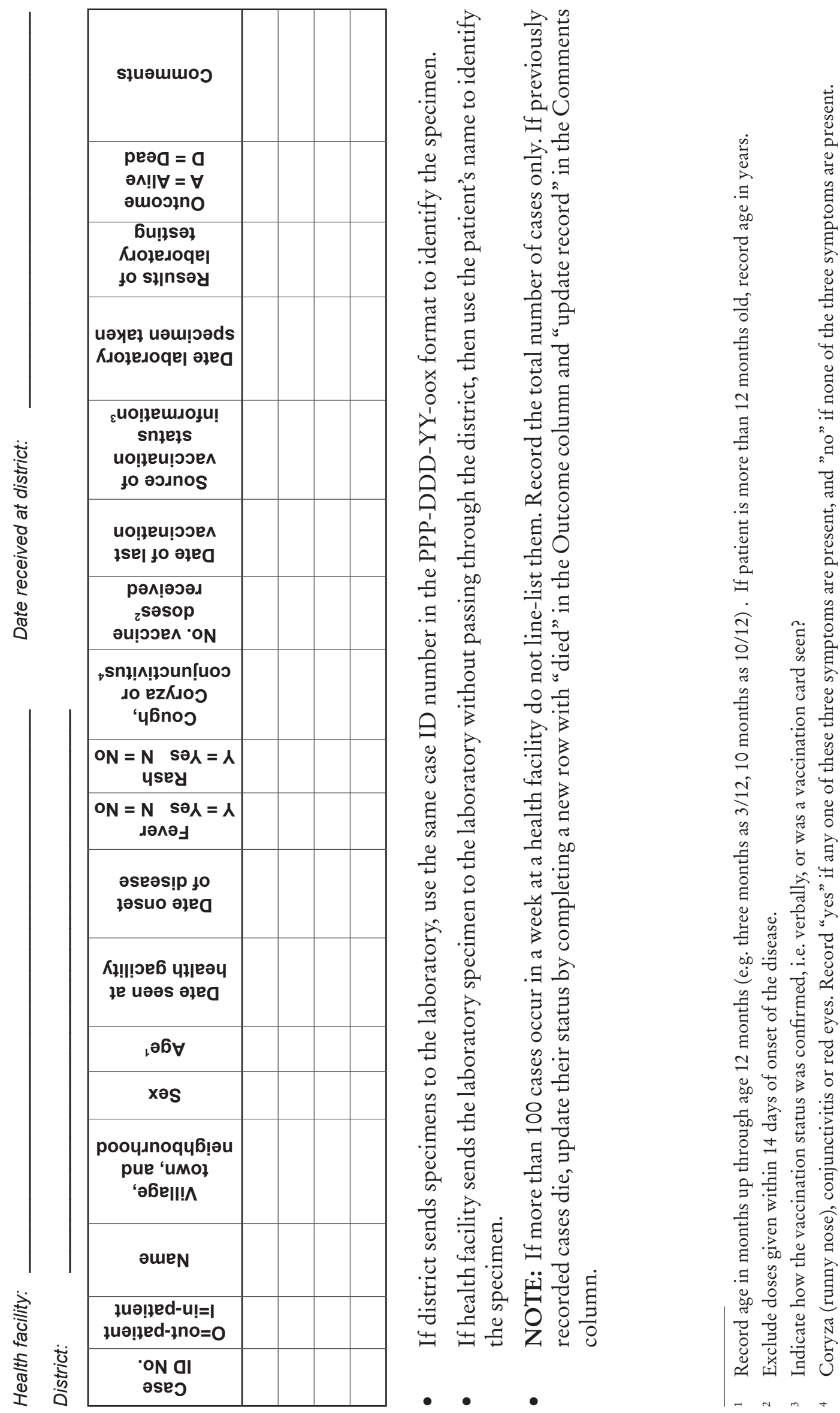


\section{Annex 5: References}

1) Guidelines for epidemic preparedness and response to measles outbreaks. Geneva, World Health Organization, 1999 (WHO/CDS/CSR/ISR/99.1).

2) Aylward RB, Clements J, Olivé JM. The impact of immunization control activities on measles outbreaks in middle and low income countries. International Journal of Epidemiology, 1997, 26(3):662-669.

3) Global Immunization Vision and Strategy. Global Immunization Vision and Strategy 2006-2015. Geneva, World Health Organization, 2005 (WHO/IVB/05.05), (http://www.who.int/vaccines/givs).

4) World Health Organization. Progress in global measles control and mortality reduction, 2000-2007. Weekly Epidemiological Record, 2008, 83:441-448.

5) International Health Regulations, 2005, to be accessed at (http://www.who.int/ gb/ebwha/pdf files/WHA58/WHA58 3-en.pdf).

6) Module on best practices for measles surveillance. Geneva, World Health Organization, 2001 (http://www.who.int/vaccines-documents/DocsPDF01/ www617.pdf, accessed November 2007).

7) Measles vaccine. WHO Position Paper. Weekly Epidemiological Record. 2004, 79:129-144 (http://www.who.int/immunization/wer7914measles April2004 position paper.pdf).

8) Cutts FT. The immunological basis for immunization: Module $N^{\circ}$ 7: Measles. Geneva, World Health Organization, 1995 (unpublished document WHO/EPI/ GEN/95.03 Rev.2).

9) WHO/UNICEF joint statement on reducing measles mortality in emergencies. Geneva, World Health Organization and United Nations Children's Fund, 2004 (http://whqlibdoc.who.int/hq/2004/WHO V\&B 04.03.pdf).

10) Pan American Health Organization. Measles elimination: field guide. Washington, DC, 2005 (http://www.paho.org/english/ad/fch/im/fieldguide measles.pdf).

11) WHO/UNICEF joint statement on global plan for reducing measles mortality 2006-2010. Geneva, World Health Organization and United Nations Children's Fund, 2006. (http://www.who.int/immunization delivery/adc/measles/ Measles\%20Global\%20Plan Eng.pdf).

12) Grais RF et al. Exploring the time to intervene with a reactive mass vaccination campaign in measles epidemics. Epidemiology and Infection, 2006, 1-5.

13) Grais RF et al. Estimating transmission intensity for a measles epidemic in Niamey, Niger: lessons for intervention. Transactions of the Royal Society of Tropical Medicine and Hygiene, 2006, 100:867-873. 
14) Guris D et al. Measles outbreaks in Micronesia 1991-1994. The Pediatric Infectious Disease Journal, 1998, 17:33-39.

15) Centers for Disease Control and Prevention. Measles Outbreak - Guam, 1994. MMWR. Morbidity and Mortality Weekly Report, 1995, 44(36):657-660.

16) Hyde TB et al. Measles outbreak in the Republic of the Marshall Islands, 2003. International Journal of Epidemiology, 2005, doi:10.1093/ije/dyi222.

17) Al Wahaibi S, El-Bushra HE, Al-Sulaiman MA. Measles outbreak in Riyadh City, 1997. Saudi Epidemiology Bulletin, 1997, 4(2):4-5.

18) Centers for Disease Control and Prevention. Emergency measles control activities - Darfur, Sudan, 2004. MMWR. Morbidity and Mortality Weekly Report, 2004, 52(38):897-899.

19) Venczel L et al. Measles eradication in the Americas: experience in Haiti. The Journal of Infectious Diseases, 2003, 187(Suppl. 1):S127-S132.

20) Centers for Disease Control and Prevention. Outbreak of measles - Venezuela and Colombia, 2001-2002. MMWR. Morbidity and Mortality Weekly Report, 2002, 51(34):757-760.

21) Soula $\mathrm{G}$ et al. Description et analyse d'une epidemie de rougeole à Bobo Dioulasso, Octobre 1995-Mai 1996 [Description and analysis of a measles epidemic in Bobo Dioulasso October 1995-May 1996]. Bulletin de Liaison et de Documentation de l'OCEAC 1998, 31(1):22-34.

22) Sniadack DH et al. Measles epidemiology and outbreak response in a rural community in Peru. Bulletin of the World Health Organization, 1999, 77(7):545-552.

23) Garly ML et al. Prophylactic antibiotics to prevent pneumonia and other complications after measles: community-based randomized double-blind placebo controlled trial in Guinea-Bissau. BMJ (Clinical Research edition), 2006, 16 December, 333(7581):1245. Epub 2006 Oct 23.

24) Guidelines for care at the first-referral level in developing countries. Geneva, World Health Organization (http://www.who.int/child-adolescent health/publications/CHILD HEALTH/WHO FCH CAH 00.1.html).

25) WHO Regional Office for Africa: Guidelines for measles surveillance (2004). Geneva, World Health Organization (http://www.afro.who.int/measles/ guidelines/measles surveillance guideline jan2006.pdf).

26) Centers for Disease Control and Prevention. Progress in Measles Control Zambia, 1999-2004. MMWR. Morbidity and Mortality Weekly Report, 2005, 54(23): 581-584.

27) WHO Regional Office For Africa: Measles supplementary immunization activities (SIAs) field guide. Geneva, World Health Organization (http://afro.who.int/ measles/guidelines/measles_sias_field_guide_revised_jan2006.pdf)

28) A further discussion on case-fatality rates can be found in WHO/EPI/GEN/93.3, Generic protocol for estimating measles case-fatality rates in a community, either during an epidemic or in a bighly endemic area. Available from WHO/EPI, Geneva, World Health Organization. 
The World Health Organization has provided technical support to its Member States in the field of vaccine-preventable diseases since 1975. The office carrying out this function at WHO headquarters is the Department of Immunization, Vaccines and Biologicals (IVB).

IVB's mission is the achievement of a world in which all people at risk are protected against vaccine-preventable diseases. The Department covers a range of activities including research and development, standard-setting, vaccine regulation and quality, vaccine supply and immunization financing, and immunization system strengthening.

These activities are carried out by three technical units: the Initiative for Vaccine Research; the Quality, Safety and Standards team; and the Expanded Programme on Immunization.

The Initiative for Vaccine Research guides, facilitates and provides a vision for worldwide vaccine and immunization technology research and development efforts. It focuses on current and emerging diseases of global public health importance, including pandemic influenza. Its main activities cover: i) research and development of key candidate vaccines; ii) implementation research to promote evidence-based decision-making on the early introduction of new vaccines; and iii) promotion of the development, evaluation and future availability of HIV, tuberculosis and malaria vaccines.
The Quality, Safety and Standards team focuses on supporting the use of vaccines, other biological products and immunizationrelated equipment that meet current international norms and standards of quality and safety. Activities cover: i) setting norms and standards and establishing reference preparation materials; ii) ensuring the use of quality vaccines and immunization equipment through prequalification activities and strengthening national regulatory authorities; and iii) monitoring, assessing and responding to immunization safety issues of global concern.

The Expanded Programme on Immunization focuses on maximizing access to high quality immunization services, accelerating disease control and linking to other health interventions that can be delivered during immunization contacts. Activities cover: i) immunization systems strengthening, including expansion of immunization services beyond the infant age group; ii) accelerated control of measles and maternal and neonatal tetanus; iii) introduction of new and underutilized vaccines; iv) vaccine supply and immunization financing; and v) disease surveillance and immunization coverage monitoring for tracking global progress.

The Director's Office directs the work of these units through oversight of immunization programme policy, planning, coordination and management. It also mobilizes resources and carries out communication, advocacy and media-related work.

\title{
Department of Immunization, Vaccines and Biologicals
}

\section{Family and Community Health}

\author{
World Health Organization \\ 20, Avenue Appia \\ $\mathrm{CH}-1211$ Geneva 27 \\ Switzerland \\ E-mail: vaccines@who.int
}

Web site: http://www.who.int/immunization/en/ 